\title{
Nonequilibrium dynamics in biomolecules
}

The celebrated concept of the relation between the structure and the function of proteins ultimately requires a microscopic understanding of the underlying conformational dynamics and the energy flow in a biomolecular system. As a major progress towards that goal, recently a number of experimental techniques have been developed which study biomolecular processes such as protein folding and molecular recognition directly and in a time-resolved manner. Rather than considering the system at equilibrium where many aspects of the dynamics and mechanism are lost due to averaging, the idea of these experiments is to prepare the system in a well-defined nonequilibrium state and monitor the time evolution transition of this nonstationary state towards equilibrium. Beautiful examples include atomic force microscopy techniques which allow us, for example, to study the molecular recognition in binding processes, as well as laser-induced $\mathrm{pH}$ and temperature-jump experiments, which may induce the folding or unfolding of a biomolecule. Aiming towards high time resolution, recently ultrafast laser experiments on photoswitchable peptides have been introduced. On the theoretical side, new simulation methods have been developed in order to describe these experiments. It is the goal of the special issue to provide a timely overview to recent experimental and theoretical progress in this new and exciting field of research.
The common theme of the first eight articles is the dynamics of protein folding. The fast initiation of these processes is either achieved by ( $\mathrm{T}-$ or $\mathrm{pH}-$ ) jump methods or by built-in photoswitches. Other articles in this section deal both with the experimental techniques and the theoretical background for the analysis of secondary structure formation. The next three articles give an impression of the current state of the art methodologies, including multidimensional vibrational spectroscopy and the calculation of infrared spectra and free energies. The four last articles provide a multi-faceted view on functional protein dynamics ranging from fast photodynamics to binding studies and molecular dynamics simulations of b-amyloid aggregation, known as the essential step in the development of Alzheimer's dementia, a widespread protein-misfolding disease.

We are grateful to the authors who contributed to this Special Issue, giving an impression of the field of conformational dynamics and also pointing out some perspectives of the future developments.

Guest Editors

G. Stock

J. Wachtveitl

H. Grubmueller

Available online 17 October 2005 